\title{
Exogenously applied growth promoters modulate the antioxidant enzyme system to improve the cotton productivity under water stress conditions
}

\author{
Nazim Hussain, Azra Yasmeen, Muhammad Ahsan Afzal \\ Department of Agronomy, Bahauddin Zakariya University, Multan, Pakistan
}

\begin{abstract}
Great climatic inconsistency and increased frequent occurrences of severe conditions results in plants being exposed to water stress at various growth stages, thus adversely affecting the productivity. This investigation was planned to minimize the water stress induced-losses to cotton plants with the exogenous application of growth promoters i.e. distilled water, salicylic acid $(0.5 \mathrm{mM})$, jasmonic acid $(50 \mu \mathrm{M})$ and moringa leaf extract (MLE30) including control (un sprayed). Cotton plants were exposed to water stress with the application of irrigation water at 10 (well-watered) and 30 day intervals (severe water stress). Results indicated that water stress severely reduced the cotton productivity. It was observed that exogenous application of salicylic acid and moringa leaf extract improved the productivity both under well-watered and water stress conditions as compared to other treatments. However, exogenous application of salicylic acid had greater influence on the studied parameters than MLE, although the insignificant differences were documented in most of the traits. Exogenous application of salicylic acid reduced the cell
\end{abstract}

Correspondence: Azra Yasmeen, Department of Agronomy, Bahauddin Zakariya University, Multan 60800, Pakistan.

E-mail: azra.yasmeen@bzu.edu.pk

Key words: Water stress; moringa leaf extract; salicylic acid; antioxidant.

Acknowledgements: this study was a part of Ph.D. thesis entitled Exploration and Modulation of abiotic stress tolerance in cotton (Gossypisum hirsutum) genotypes. The Authors are thankful to the Department of Agronomy, Bahauddin Zakariya University Multan, for provision of research facilities for experimentation.

Contributions: NH and MAA conceived and designed the experiment; AY analyzed the data; AY and MAA contributed materials/analysis tools; MAA wrote the paper.

Conflicts of interest: the authors declare no conflict of interest.

Received for publication: 9 September 2019.

Revision received: 5 February 2020.

Accepted for publication: 9 March 2020.

(C) Copyright: the Author(s), 2020

Licensee PAGEPress, Italy

Italian Journal of Agronomy 2020; 15:1537

doi:10.4081/ija.2020.1537

This article is distributed under the terms of the Creative Commons Attribution Noncommercial License (by-nc 4.0) which permits any noncommercial use, distribution, and reproduction in any medium, provided the original author(s) and source are credited. injury percentage, improved the cell membrane thermostability and produced significantly higher content of leaf protein, superoxide dismutase (SOD), peroxidase (POD) and catalase (CAT) that modulated the negative influence of water stress on yield contributing attributes and produced maximum seed cotton yield. These results indicate that exogenous application of SA and MLE helped the cotton plants to become more tolerant to water stressinduced losses by adjusting the membrane characteristics and improving their antioxidant defence mechanism.

\section{Introduction}

Cotton growth and productivity is adversely affected by abiotic stresses such as salinity, flooding, high temperature and water stress (Saifuddin et al., 2016). Out of these factors, water stress is one of the key threats to plants that leads to modifications at all levels from ecological, morphological, physiological, biochemical and molecular characteristics of plants (Muscolo et al., 2014). Water stress at vegetative stage reduced the plant growth and development that resulting in the reduction of root and shoot length and their fresh and dry biomass, therefore roots and shoots may become thinner or thicker (Ashraf and Foolad, 2007). However, cotton plants are highly sensitive to water stress during flowering and boll formation stage that results in slow growth and increase the shedding rate of flowers and bolls (Kawakami et al., 2010; Isokpehi et al., 2011). Cotton seed yield was also reduced with the decline in total number of bolls per plant under water stress (Hamada, 2000; Golldack et al., 2014).

Adverse impacts of water stress on cotton plants can be reduced with the exogenous application of plant growth promoters (Farooq et al., 2009). Salicylic acid (SA) is a naturally occurring plant hormone working as a vital signaling molecule with pervasive distribution in plants and contributes in numerous plant physiology processes (Clarke et al., 2000). SA plays fundamental roles in plants including seed germination, growth, respiration, stomatal movement, cell division, cell elongation, sink/source regulation, photosynthetic activity and protein synthesis of the crop plants (Arfan et al., 2007). Exogenous application of SA to water stressed plants diminishes the cell membrane damage and improves the activities of antioxidant enzymes such as SOD, CAT and POD in leaves (Khan et al., 2014).

Another class of phytohormones, Jasmonic acid (JA) is an endogenous regulator derived from the metabolism of fatty acids membrane. It plays significant role in controlling stress reactions, plant growth and development in crested wheat grass (Shan and Liang, 2010). The primary function of JA under water stress condition is to avoid water loss to sustain cell turgor and water gradient uptake into the cell. Besides osmoregulation function, compatible solutes protect enzymes, scavenge free oxygen radicals and protect membrane structures and integrity (Ashraf and Foolad, 2007). JA responsible for regulation of numerous physiological 
and metabolic processes in plants such as vegetative growth, stamen and trichome development, regulates anthocyanin production, senescence and fruit settings (Yoshida et al., 2009).

Exogenous application of these plant growth promoters is the most effective approach involved in promoting plant growth and development by minimizing or alleviating the adverse effects of water stress on the plant growth and metabolism in different crops (Sadak and Dawood, 2014), but this application is expensive. Thus, there is continuous need to search for alternative safe natural sources containing nutrients, hormones and antioxidants with higher economic returns (Yasmeen et al., 2018). Moringa oleifera L. is one of such substitute, being explored to establish its influence on growth and productivity of wheat under stress conditions (Yasmeen et al., 2013). Moringa leaf extract (MLE) had extensively been used to improve the stress tolerance in quinoa and broad beans plants by causing modulations in biochemistry and plant physiology required for stress tolerance (Ismail et al., 2016). It is massive source of zeatin (a purine adenine derivative of plant hormone group cytokinin), riboflavin, vitamin $\mathrm{A}$ and $\mathrm{C}$, phenols, mineral nutrients, antioxidant and some osmoprotectant that making it a potential bio-stimulant (Arif et al., 2019). Foliar spray of MLE has positive influences on the wheat plant growth, rate of photosynthesis, hormonal content and anti-oxidative resistance activities in stress conditions (Yasmeen et al., 2013). Exogenously applied MLE improved the antioxidant system, stimulated plant defence system, enhanced the levels of plant secondary metabolites and boosting the Phaseolus vulgaris L. (Rady and Mohamed, 2015) and rice performance under water stress conditions (Rehman et al., 2015).

Although a lot of work has been done on different crops to mitigate the adverse effects of water stress by exogenous application of above studied growth promoters. However, only a limited studies showing the potential of salicylic acid, jasmonic acid and morinag leaf extract to promote the growth and productivity of cotton under water stress conditions are available. Therefore, we hypothesized that the foliar application of selected growth promoters contributes towards improving the physiological and antioxidant attributes and enhance the cotton productivity under water stress conditions. Keeping in view the above-mentioned discussion, the present two years study was planned to investigate the efficiency of exogenous application of distilled water, SA, JA and MLE in mitigating the adverse impacts of water stress on cotton productivity, so as to explore their practical and sustainable agricultural applications.

\section{Materials and methods}

A field investigation was planned at agronomic research area of Bahauddin Zakariya University, Multan, Pakistan $\left(30.2639^{\circ} \mathrm{N}\right.$, $71.5101{ }^{\circ} \mathrm{E}$; $123 \mathrm{~m}$ above sea level) during 2014 and 2015 to explore the role of plant growth promoters in mitigating the adverse impacts of water stress on $B t$ cotton productivity. This trial was designed in a completely randomized block with factorial arrangement having three replications. The net plot size was $5.0 \times 3.0 \mathrm{~m}$ comprised of 4 rows and each row $75 \mathrm{~cm}$ apart in each experimental unit. The treatments included two irrigation intervals i.e., irrigation at 10 (well-watered) and 30 day intervals (water stressed) and four exogenous application of growth promoters i.e. distilled water (Arif et al., 2019), salicylic acid (0.5 mM) (Khan et al., 2014), jasmonic acid (50 $\mu \mathrm{M})$ (Yosefi et al., 2018) and moringa leaf extract (MLE30) (Yasmeen et al., 2016) including control (no foliar spray, only soil irrigations were applied according to treatments). Foliar sprays of these growth promoters were applied once at $50 \%$ vegetative growth (60 days after sowing), flowering (90 days after sowing) and boll development stages (120 days after sowing) with flat fan nozzle.

\section{Preparation of moringa leaf extract}

Moringa leaves extract (MLE) is potential source of zeatin that enhances the antioxidant properties of many enzymes and protects the cells from injuries (Zhang and Ervin, 2004). Young leaves of Moringa oleifera plants were harvested and washed several times with distilled water then stored in freezer at $-5{ }^{\circ} \mathrm{C}$ for $12 \mathrm{~h}$. Moringa frozen leaves were crushed in a juicer machine for extraction according to the procedure explained by Yasmeen et al. (2018). The extract was filtered twice by using Whatman No.1 filter paper and then centrifuged at $8000 \mathrm{~g}$ for 20 minutes and diluted 30 times with distilled water.

\section{Crop husbandry}

Pre-soaking irrigation $(15 \mathrm{~cm})$ was applied before land preparation of experimental area. A fine seedbed was prepared by cultivating the experimental area twice followed by planking and bed shaper was used for suitable beds preparation. Seeds of cotton cultivar FH-142 were dibbled manually during first week of June 2014 and 2015. Planting was processed, keeping $75 \mathrm{~cm}$ row-torow and $30 \mathrm{~cm}$ plant-to-plant distance. To ensure successful seed germination and emergence, the furrows were irrigated $72 \mathrm{~h}$ after planting. Rests of the irrigations water were applied according to the treatments. Recommended dose of nitrogen fertilizer at $145 \mathrm{~kg}$ $\mathrm{ha}^{-1}$ was broadcasted in three equal parts in the form of Urea at distinctive growth stages i.e. sowing time, beginning of bloom and peak blooming stage. Whereas, recommended dose of phosphorus $\left(56 \mathrm{~kg} \mathrm{ha}^{-1}\right)$ and potassium fertilizer $\left(62 \mathrm{~kg} \mathrm{ha}^{-1}\right)$ was broadcasted in the form of single super phosphate and sulfate of potash at sowing time, respectively. Weeds were controlled with the application of pre-emergence herbicide of Pendimethalin (Stomp-330E at 2.5 $\mathrm{L} \mathrm{ha} \mathrm{a}^{-1}$ ), with four-hand weeding at the $8^{\text {th }}, 15^{\text {th }}, 22^{\text {nd }}$ and $29^{\text {th }}$ days after sowing. To keep cotton crop under the threshold level, different plant protection treatments were applied. All farming practices except experimental treatments were kept similar for each plot.

\section{Data collection}

After 25 days of sowing, 20 randomly selected plants were tagged from each experimental unit to observe the sympodial branches, number of bolls and mean boll weight. Manual harvesting of seed cotton was performed twice in the middle two rows. Two cotton pickings were made during third week of October and first week of December during both growing seasons. Ten days after every exogenous application of growth promoters, ten leaves sample were taken from each experimental unit to observe the antioxidant enzyme activities. All the leaves samples freeze, dried and then $0.5 \mathrm{~g}$ powder taken from freeze-dried leaves sampled obtained previously selected tagged plants were homogenized with $50 \mathrm{mM} \mathrm{Na}_{2} \mathrm{HPO}_{4}-\mathrm{NaH}_{2} \mathrm{PO}_{4}$ buffer containing $0.2 \mathrm{mM}$ EDTA and $2 \%$ insoluble polyvinyl pyrrolidone in a chilled pestle and mortar. The slurry was centrifuged at $12,000 \times \mathrm{g}$ for $20 \mathrm{~min}$ and the supernatant was used for enzyme activities assay. Standard protocols were adopted to measure peroxidase (POD), catalase (CAT) (Chance and Maehly, 1955), superoxide dismutase (SOD) (Giannopolitis and Reis, 1997) and total phenolic contents (Waterhouse, 2001). Cell membrane thermostability (CMT) was calculated from RCI\%. Sullivan (1972) developed a heat tolerant 
test that determines relative cell injury percentage ( $\mathrm{RCI} \%$ ) through measuring the amount of electrolyte leakage from leaf disks bathed in deionized water after exposure to heat treatment. By using punch machine, round leaf discs of $10 \mathrm{~mm}$ in diameter were made after removing fresh cotton leaves. Discs were put in the glass vials having $2 \mathrm{~mL}$ deionized water and washed, thereafter, $2 \mathrm{~mL}$ double distilled water was added to each vial and capped cotton plug. One set of tubes was placed at $50^{\circ} \mathrm{C}$ for one hour in a water bath, while the other set (control) was placed at $25^{\circ} \mathrm{C}$ for the same time. After this treatment, vials were kept at $10^{\circ} \mathrm{C}$ for $24 \mathrm{~h}$ and then brought to $25^{\circ} \mathrm{C}$. Electrical conductivity (EC) meter was used to measure EC of these samples. In order to kill the tissues and release all electrolytes, vials were autoclaved for 10 minutes at $10 \mathrm{MPa}$. Final EC was again measured and used the formula given by Sullivan (1972), to calculate relative cell injury $(\mathrm{RCI})$ :

RCI $(\%)=1-\left[\left\{1-\left(\mathrm{T}_{1} / \mathrm{T}_{2}\right)\right\} /\left\{1-\left(\mathrm{C}_{1} / \mathrm{C}_{2}\right)\right\}\right] \times 100$ where, $\mathrm{T}_{1}=$ initial $\mathrm{EC}$ value of heat-treated vial, $\mathrm{C}_{1}=$ initial $\mathrm{EC}$ value of control vial, $\mathrm{T}_{2}=$ final $\mathrm{EC}$ value of heat-treated vial, $\mathrm{C}_{2}=$ final EC value of control vial.

\section{Statistical procedure}

All data collected were subjected to the analysis of variance (ANOVA) by using computer-based software MSTAT C. Means of the different treatments were compared by adopting DMR test at $5 \%$ level of probability (Steel et al., 1997).

\section{Results}

\section{Yield and yield contributing characteristics}

Sympodial branches are the bolls bearing branches for producing seed cotton. Foliar spray of MLE and salicylic acid to wellwatered plants produced maximum sympodial branches per plant (16.14 and 14.81) during first and second growing season, respectively (Table 1$)$. While water stressed plants produced minimum sympodial branches per plant (7.76 and 7.33).

Number of bolls on cotton plant mounts ups the extent of seed cotton per plant that consequently hoards the seed cotton yield per unit area. Foliar spray of salicylic acid to well-watered plants pro- duced maximum bolls per plant (17.07 and 15.81) during first and second growing season, respectively (Table 1). While water stressed plants produced minimum bolls per plant (6.63 and 5.89).

Foliar spray of MLE to well-watered plants produced maximum boll weight (3.42 and $3.59 \mathrm{~g}$ ) during first and second growing season, respectively (Table 1). While water stressed plants produced minimum boll weight (2.83 and $2.86 \mathrm{~g}$ ). Statistical analysis of data revealed significant effect on interaction between foliar treatments and irrigation intervals was significant during 2014 and 2015 (Table 1).

Foliar spray of salicylic acid to well-watered plants produced maximum seed cotton yield (2323.8 and $2250.4 \mathrm{~kg} \mathrm{ha}^{-1}$ ) during first and second growing season, respectively (Table 1). While water stressed plants produced minimum seed cotton yield (745.3 and $672.2 \mathrm{~kg} \mathrm{ha}^{-1}$ ).

\section{Biochemical characteristics}

Relative cell injury percentage is a marker of cellular membrane thermostability. Maximum relative cell injury percentage (49.49 and 51.49\%) was produced from water stressed plant in control plots during 2014 and 2015. While foliar application of MLE to well-watered plants produced minimum RCI (39.15 and $41.82 \%$ ). Statistical analysis of variance showed significant effects on interaction between foliar treatments and irrigation intervals was significant during 2014 and 2015 (Table 2).

Cell membrane thermo stability was recorded indirectly through assessing RCI that exposes the relative electrical conductivity (REC) i.e., the comparative volume of electrolytes leaked out due to injury in cell membranes instigated by heat shock. Foliar spray of MLE to well-watered plants produced significantly higher cell membrane thermo stability (30.50 and 32.83) during first and second growing season, respectively (Table 2). While water stressed plants produced minimum cell membrane thermo stability (22.02 and 24.02).

Foliar spray of MLE and salicylic acid to well-watered plants produced significantly higher leaf total soluble protein $(24.98$ and $24.43 \mathrm{mg} \mathrm{g}^{-1}$ ) during first and second growing season, respectively (Table 2 ). While water stressed plants produced minimum leaf total soluble protein (16.45 and $15.88 \mathrm{mg} \mathrm{g}^{-1}$ ) during both growing seasons.

Table 1. Influence of growth promoters on the sympodial branches, bolls harvested, mean boll weight ( $\mathrm{g}$ ) and seed cotton yield (kg ha $\left.{ }^{-1}\right)$ contributing attributes of cotton.

\begin{tabular}{|c|c|c|c|c|c|c|c|c|c|}
\hline \multirow[t]{2}{*}{ Water level } & \multirow[t]{2}{*}{$\begin{array}{l}\text { Foliar spray of } \\
\text { growth promoters }\end{array}$} & \multicolumn{2}{|c|}{$\begin{array}{l}\text { Sympodial } \\
\text { branches }\end{array}$} & \multicolumn{2}{|c|}{$\begin{array}{c}\text { Bolls } \\
\text { harvested }\end{array}$} & \multicolumn{2}{|c|}{$\begin{array}{l}\text { Mean boll } \\
\text { weight (g) }\end{array}$} & \multicolumn{2}{|c|}{$\begin{array}{l}\text { Seed cotton yield } \\
\left(\mathrm{kg} \mathrm{ha}^{-1}\right)\end{array}$} \\
\hline & & 2014 & 2015 & 2014 & 2015 & 2014 & 2015 & 2014 & 2015 \\
\hline \multirow[t]{5}{*}{ Well-watered } & Control & $12.44^{\mathrm{c}}$ & $12.10^{\mathrm{c}}$ & $13.72^{\mathrm{c}}$ & $12.39^{c}$ & $3.24^{\mathrm{ac}}$ & $3.37^{\mathrm{a}}$ & $1775.3^{\mathrm{c}}$ & $1670.8^{b}$ \\
\hline & Distilled water & $13.80^{\mathrm{bc}}$ & $12.87^{\mathrm{bc}}$ & $14.75^{\mathrm{bc}}$ & $13.38^{\mathrm{bc}}$ & $3.30^{\mathrm{ab}}$ & $3.44^{\mathrm{a}}$ & $1947.6^{\mathrm{c}}$ & $1843.5^{b}$ \\
\hline & Salicylic acid & $16.14^{\mathrm{a}}$ & $14.81^{\mathrm{a}}$ & $17.07^{\mathrm{a}}$ & $15.81^{\mathrm{a}}$ & $3.40^{\mathrm{a}}$ & $3.56^{\mathrm{a}}$ & $2323.8^{\mathrm{a}}$ & $2250.4^{\mathrm{a}}$ \\
\hline & Jasmonic acid & $14.61^{\mathrm{ab}}$ & $13.17^{\mathrm{bc}}$ & $15.27^{\mathrm{ac}}$ & $13.61^{b c}$ & $3.32^{\mathrm{ab}}$ & $3.46^{\mathrm{a}}$ & $2026.5^{b c}$ & $1881.8^{b}$ \\
\hline & Moringa leaf extract & $15.44^{\mathrm{ab}}$ & $14.30^{\mathrm{ab}}$ & $16.48^{\mathrm{ab}}$ & $14.98^{\mathrm{ab}}$ & $3.42^{\mathrm{a}}$ & $3.59^{\mathrm{a}}$ & $2246.7^{\mathrm{ab}}$ & $2142.3^{\mathrm{a}}$ \\
\hline \multirow[t]{5}{*}{ Irrigation at 30 days interval } & Control & $7.76^{\mathrm{d}}$ & $7.33^{\mathrm{d}}$ & $6.63^{\mathrm{e}}$ & $5.89^{\mathrm{e}}$ & $2.83^{\mathrm{d}}$ & $2.86^{\mathrm{b}}$ & $745.3^{\mathrm{f}}$ & $672.2^{\mathrm{d}}$ \\
\hline & Distilled water & $8.19^{d}$ & $7.83^{\mathrm{d}}$ & $7.07^{\mathrm{e}}$ & $6.21^{\mathrm{e}}$ & $2.93^{\mathrm{cd}}$ & $2.90^{\mathrm{b}}$ & $834.3^{\mathrm{ef}}$ & $718.4^{\mathrm{d}}$ \\
\hline & Salicylic acid & $9.30^{\mathrm{d}}$ & $8.63^{\mathrm{d}}$ & $9.16^{\mathrm{d}}$ & $8.50^{\mathrm{d}}$ & $3.05^{\text {bd }}$ & $3.03^{b}$ & $1117.0^{\mathrm{d}}$ & $1028.7^{c}$ \\
\hline & Jasmonic acid & $8.61^{\mathrm{d}}$ & $7.94^{\mathrm{d}}$ & $8.09^{\mathrm{de}}$ & $6.39^{\mathrm{e}}$ & $2.95^{\mathrm{cd}}$ & $2.97^{\mathrm{b}}$ & $958.1^{\mathrm{df}}$ & $757.2^{\mathrm{d}}$ \\
\hline & Moringa leaf extract & $8.82^{\mathrm{d}}$ & $8.15^{\mathrm{d}}$ & $9.03^{\mathrm{d}}$ & $8.30^{\mathrm{d}}$ & $3.05^{\mathrm{bc}}$ & $3.02^{\mathrm{b}}$ & $1100.6^{\text {de }}$ & $997.6^{\mathrm{c}}$ \\
\hline \multicolumn{2}{|c|}{ LSD $0.05 p$} & 1.6352 & 1.5166 & 1.8979 & 1.7980 & 0.3226 & 0.3057 & 281.26 & 234.47 \\
\hline
\end{tabular}

\footnotetext{
a-f Means followed by same letter(s) are not significantly different at $\mathrm{P}<0.05$.
} 
Foliar spray of MLE to well-watered plants produced maximum total phenolic contents (14.67 and $14.31 \mathrm{mg} \mathrm{g}^{-1}$ ) during first and second growing season, respectively. While water stressed plants produced minimum total phenolic contents (11.11 and 11.34 $\left.\mathrm{mg} \mathrm{g}^{-1}\right)$.

Under water stress condition, foliar spray of MLE produced significantly higher superoxide dismutase (65.04 IU min ${ }^{-1} \mathrm{mg}$ protein $^{-1}$ ) during first growing season (Table 3). While foliar application of distilled water on well-watered plants produced minimum superoxide dismutase (54.53 IU min $\mathrm{mg}^{-1} \mathrm{mrotein}^{-1}$ ).

Under water stress condition, foliar spray of MLE produced significantly higher peroxidase (6.56 and $\left.6.46 \mathrm{mmol} \mathrm{min}^{-1} \mathrm{mg}_{\text {protein }}^{-1}\right)$ during first and second growing season, respectively (Table 3 ). While well-watered plants produced minimum peroxidase (5.23 and 5.41 mmol $\mathrm{min}^{-1} \mathrm{mg}$ protein ${ }^{-1}$ ) with the exogenous application of distilled water. Statistical analysis of variance showed significant variations between foliar treatments and irrigation interval was significant during 2014 and 2015 (Table 3).

Foliar spray of MLE to water stressed plants produced significantly higher catalase $\left(77.76 \mathrm{mmol} \mathrm{min}^{-1} \mathrm{mg}\right.$ protein $\left.{ }^{-1}\right)$ during 2015 (Table 3). While well-watered plants produced minimum catalase $\left(70.95 \mathrm{mmol} \mathrm{min}^{-1} \mathrm{mg}\right.$ protein $\left.{ }^{-1}\right)$.

\section{Discussion}

Water stress has been stated as one of the severe abiotic stresses currently influencing agricultural crops. In present study the executed water stress adversely affect nearly all the morphological and physiological attributes of cotton plants, which ultimately reduced the productivity (Anjum et al., 2014). A sympodial branch in cotton is one of the key factors influencing the cotton productivity (Bibi et al., 2010). In the present study, maximum number of sympodial branches per plant was observed in well-watered conditions against the minimum were noted under water stress conditions. Presence of considerable variation in cotton for sympodial branches in response to water stress was also observed by Ratnakumari and Subbaramamma (2006) and Sahito et al. (2015). Foliar application of salicylic acid produced significantly higher number of sympodial branches per plant that suggest the role of salicylic acid in improving cotton reproductive growth under water stress conditions (Noreen et al., 2015).

Bolls is an essential attribute, which directly interrelated to cotton productivity (Bibi et al., 2010). In this study water stress had negative effect on the total number of bolls per plant and mean boll weight. The LSD at (0.05) probability was computed to bring

Table 2. Influence of growth promoters on the RCI\%, CMT, Leaf soluble protein $\left(\mathrm{mg}^{-1}\right)$ and phenolic contents (mg $\left.\mathrm{g}^{-1}\right)$ attributes of cotton.

\begin{tabular}{|c|c|c|c|c|c|c|c|c|c|}
\hline \multirow[t]{2}{*}{ Water level } & \multirow{2}{*}{$\begin{array}{l}\text { Foliar spray } \\
\text { of growth } \\
\text { promoters }\end{array}$} & \multicolumn{2}{|c|}{$\begin{array}{l}\text { Relative cell } \\
\text { injury percentage }\end{array}$} & \multicolumn{2}{|c|}{$\begin{array}{l}\text { Cell membrane } \\
\text { thermostability }\end{array}$} & \multicolumn{2}{|c|}{$\begin{array}{l}\text { Leaf soluble protein } \\
\qquad\left(\mathrm{mg} \mathrm{g}^{-1}\right)\end{array}$} & \multicolumn{2}{|c|}{$\begin{array}{l}\text { Phenolic contents } \\
\qquad\left(\mathrm{mg} \mathrm{g} \mathrm{g}^{-1}\right)\end{array}$} \\
\hline & & 2014 & 2015 & 2014 & 2015 & 2014 & 2015 & 2014 & 2015 \\
\hline Well-watered & $\begin{array}{l}\text { Control } \\
\text { Distilled water } \\
\text { Salicylic acid } \\
\text { Jasmonic acid } \\
\text { Moringa leaf extract }\end{array}$ & $\begin{array}{l}42.72^{\mathrm{bc}} \\
42.56^{\mathrm{bc}} \\
39.15^{\mathrm{c}} \\
41.20^{\mathrm{bc}} \\
40.48^{\mathrm{c}}\end{array}$ & $\begin{array}{l}46.05^{\text {be }} \\
44.23^{\text {be }} \\
41.82^{\mathrm{e}} \\
43.54^{\text {ce }} \\
42.15^{\text {de }}\end{array}$ & $\begin{array}{l}25.48^{\mathrm{ac}} \\
26.34^{\mathrm{ac}} \\
30.50^{\mathrm{a}} \\
27.52^{\mathrm{ac}} \\
29.03^{\mathrm{ab}}\end{array}$ & $\begin{array}{l}28.14^{\mathrm{bd}} \\
29.00^{\mathrm{bc}} \\
32.70^{\mathrm{a}} \\
30.39^{\mathrm{ab}} \\
32.83^{\mathrm{a}}\end{array}$ & $\begin{array}{l}19.04^{\mathrm{bc}} \\
21.43^{\mathrm{ab}} \\
24.77^{\mathrm{a}} \\
23.03^{\mathrm{a}} \\
24.98^{\mathrm{a}}\end{array}$ & $\begin{array}{l}18.37^{\mathrm{cd}} \\
20.66^{\mathrm{bc}} \\
24.43^{\mathrm{a}} \\
22.69^{\mathrm{ab}} \\
23.31^{\mathrm{ab}}\end{array}$ & $\begin{array}{l}12.61^{\mathrm{cd}} \\
12.90^{\mathrm{bd}} \\
13.83^{\mathrm{ab}} \\
13.33^{\mathrm{bc}} \\
14.67^{\mathrm{a}}\end{array}$ & $\begin{array}{r}12.94^{\mathrm{ac}} \\
13.23^{\mathrm{ab}} \\
14.16^{\mathrm{a}} \\
13.59^{\mathrm{ab}} \\
14.31^{\mathrm{a}}\end{array}$ \\
\hline Irrigation at 30 days interval & $\begin{array}{l}\text { Control } \\
\text { Distilled water } \\
\text { Salicylic acid } \\
\text { Jasmonic acid } \\
\text { Moringa leaf extract }\end{array}$ & $\begin{array}{l}49.49^{\mathrm{a}} \\
47.28^{\mathrm{ab}} \\
45.49^{\mathrm{ac}} \\
47.46^{\mathrm{ab}} \\
44.73^{\mathrm{ac}}\end{array}$ & $\begin{array}{l}51.49^{\mathrm{a}} \\
48.95^{\mathrm{ac}} \\
47.49^{\mathrm{ad}} \\
49.46^{\mathrm{ab}} \\
46.07^{\mathrm{be}}\end{array}$ & $\begin{array}{l}22.02^{\mathrm{c}} \\
22.61^{\mathrm{bc}} \\
23.16^{\mathrm{bc}} \\
22.82^{\mathrm{bc}} \\
23.57^{\mathrm{bc}}\end{array}$ & $\begin{array}{l}24.02^{\mathrm{e}} \\
25.61 \mathrm{~d}^{\mathrm{e}} \\
26.19^{\mathrm{ce}} \\
25.52^{\mathrm{de}} \\
26.57^{\mathrm{ce}}\end{array}$ & $\begin{array}{l}16.45^{\mathrm{c}} \\
16.71^{\mathrm{c}} \\
18.74^{\mathrm{bc}} \\
18.10^{\mathrm{bc}} \\
18.43^{\mathrm{bc}}\end{array}$ & $\begin{array}{l}15.88^{\mathrm{d}} \\
16.02^{\mathrm{d}} \\
18.08^{\mathrm{cd}} \\
17.44^{\mathrm{d}} \\
17.76^{\mathrm{d}}\end{array}$ & $\begin{array}{l}11.11^{\mathrm{e}} \\
11.31^{\mathrm{e}} \\
12.89^{\mathrm{bd}} \\
12.12^{\mathrm{de}} \\
12.98^{\mathrm{bd}}\end{array}$ & $\begin{array}{l}11.34^{\mathrm{d}} \\
11.51^{\mathrm{d}} \\
12.55^{\mathrm{bd}} \\
11.78^{\mathrm{cd}} \\
12.65^{\mathrm{bd}}\end{array}$ \\
\hline LSD & $.05 p$ & 6.5779 & 5.4191 & 6.6476 & 3.2611 & 3.9314 & 2.7934 & 1.0808 & 1.4136 \\
\hline
\end{tabular}

a-e Means followed by same letter(s) are not significantly different at $\mathrm{P}<0.05$.

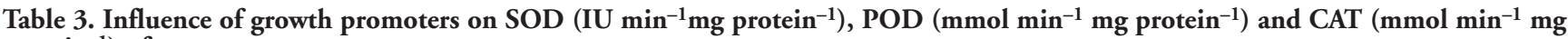
protein $^{-1}$ ) of cotton.

\begin{tabular}{|c|c|c|c|c|c|c|c|}
\hline Water level & $\begin{array}{l}\text { Foliar spray } \\
\text { of growth } \\
\text { promoters }\end{array}$ & \multicolumn{2}{|c|}{$\begin{array}{l}\text { Superoxide dismutase } \\
\left(\text { IU } \text { min }^{-1} \mathrm{mg} \mathrm{protein}^{-1}\right)\end{array}$} & \multicolumn{2}{|c|}{$\begin{array}{c}\text { Peroxidase } \\
\left(\mathrm{mmol} \mathrm{min}^{-1} \mathrm{mg} \mathrm{protein}^{-1}\right)\end{array}$} & \multicolumn{2}{|c|}{$\begin{array}{c}\text { Catalase } \\
\left(\mathrm{mmol} \mathrm{min}^{-1} \mathrm{mg}^{2} \text { protein }\right. \\
\end{array}$} \\
\hline Well-watered & $\begin{array}{l}\text { Control } \\
\text { Distilled water } \\
\text { Salicylic acid } \\
\text { Jasmonic acid } \\
\text { Moringa leaf extract }\end{array}$ & $\begin{array}{l}55.05^{\mathrm{bc}} \\
54.53^{\mathrm{c}} \\
63.36^{\mathrm{ab}} \\
60.66^{\mathrm{ac}} \\
64.69^{\mathrm{a}}\end{array}$ & $\begin{array}{l}57.38 \\
57.86 \\
61.36 \\
59.00 \\
62.02\end{array}$ & $\begin{array}{l}5.28^{\mathrm{c}} \\
5.23^{\mathrm{c}} \\
5.96^{\mathrm{b}} \\
5.91^{\mathrm{b}} \\
6.17^{\mathrm{ab}}\end{array}$ & $\begin{array}{c}5.41^{\mathrm{c}} \\
5.57^{\mathrm{bc}} \\
6.13^{\mathrm{a}} \\
5.97^{\mathrm{ab}} \\
6.41^{\mathrm{a}}\end{array}$ & $\begin{array}{l}66.28 \\
67.32 \\
72.65 \\
69.81 \\
73.68\end{array}$ & $\begin{array}{l}70.95^{\mathrm{b}} \\
71.32^{\mathrm{ab}} \\
74.00^{\mathrm{ab}} \\
72.14^{\mathrm{ab}} \\
75.34^{\mathrm{ab}}\end{array}$ \\
\hline Irrigation at 30 days interval & $\begin{array}{l}\text { Control } \\
\text { Distilled water } \\
\text { Salicylic acid } \\
\text { Jasmonic acid } \\
\text { Moringa leaf extract }\end{array}$ & $\begin{array}{l}59.82^{\mathrm{ac}} \\
58.21^{\mathrm{ac}} \\
65.04^{\mathrm{a}} \\
62.13^{\mathrm{ac}} \\
64.21^{\mathrm{a}}\end{array}$ & $\begin{array}{l}58.73 \\
59.87 \\
61.91 \\
59.89 \\
62.37\end{array}$ & $\begin{array}{c}6.17^{\mathrm{ab}} \\
5.86^{\mathrm{b}} \\
6.56^{\mathrm{a}} \\
6.24^{\mathrm{ab}} \\
6.43^{\mathrm{a}}\end{array}$ & $\begin{array}{l}6.00^{\mathrm{ab}} \\
6.19^{\mathrm{a}} \\
6.30^{\mathrm{a}} \\
6.08^{\mathrm{a}} \\
6.46^{\mathrm{a}}\end{array}$ & $\begin{array}{l}70.09 \\
71.14 \\
73.59 \\
72.34 \\
74.42\end{array}$ & $\begin{array}{l}72.42^{\mathrm{ab}} \\
73.61^{\mathrm{ab}} \\
77.76^{\mathrm{a}} \\
74.34^{\mathrm{ab}} \\
76.92^{\mathrm{ab}}\end{array}$ \\
\hline \multicolumn{2}{|c|}{ LSD $0.05 p$} & 8.4313 & n.s & 0.3977 & 0.4962 & n.s & 6.5324 \\
\hline
\end{tabular}

a-c Means followed by same letter(s) are not significantly different at $\mathrm{P}<0.05$. 
the mean differences more clear. Application of irrigation water at 10 day intervals produced maximum number of bolls per plant against the minimum was observed with the application of irrigation water at 30 day intervals. It might be due to the fact that bolls are greatly dependent on pre-flowering reserved photo-assimilate and water stress at flower and boll formation stage adversely affected the number and size of bolls. It is also possible that the considerable reduction in bolls per plant in water stress conditions can be associated with lower assimilate production during photosynthesis and its disturbance in the allocation of flowing and reserved photo-assimilates to bolls (Veesar et al., 2018). Foliar spray of salicylic acid prompted significant increase in bolls retention under water stress situations. It might be due to the fact that foliar spray of SA improved the production of antioxidant enzymes and osmolytes in higher amount triggered the cotton crop to survive the stressful circumstances through quick translocation of photo-assimilates from vegetative to reproductive structures (Arfan et al., 2007). Reduction in mean boll weight under water stress conditions might be associated with the minimum level of carbohydrates reserved in vegetative organs before fertilization (Borra's et al., 2003). Bolls treated with growth promoters have greater photosynthetic sinks for carbohydrates and other metabolites that improved the boll settings percentage and mean boll weight (Güllüoglu, 2004; Ren et al., 2013). The existence of cytokinin in MLE assist in yielding more and superior bolls (Arif et al., 2019).

Lower number of sympodial branches, bolls per plant and mean boll weight under water stressed conditions ultimately reduced the cotton productivity, which could be due to the fact that yield contributing attributes are dependent on the availability of soil moisture and antioxidant enzymes activities (Farooq et al., 2009; Korres et al., 2016). However, exogenously applied salicylic acid and MLE considerably improved the yield and yield contributing attributes that showed promising effect of these substances on several physiological processes (Wutipraditkul et al., 2015). As foliar spray of growth promoters improved the rate of photosynthesis, contributing to photo-assimilates flow toward bolls to increase carbohydrate provision during reproductive stage (Blaise et al., 2009). It was observed that foliar spray of macro and micronutrients particularly MLE was found promising to enhance flowering, bolls setting percentage and seed cotton yield (Arif et al., 2019). This rise might also be due to presence of zeatin in MLE that improves the antioxidant properties of various enzymes and protects the cell under adverse conditions (Zhang and Ervin, 2004).

$\mathrm{RCI} \%$ is a consistent feature for the screening of water stress tolerant plant. Under well-watered condition, plant cell membranes remain intact, however under water stress condition, the membranes of plant cells are damaged that leads to the release of cell content. Therefore the present results indicated the lower value of $\mathrm{RCI} \%$ in well-watered condition than water stress condition (Akbar and Hussain, 2019). In response to water stress, cotton plant increased the production of phenolic compounds (SánchezRodríguez and Rubio-Wilhelmi, 2010). Thus, phenolic compounds provide important physiological and ecological duties, being mainly involved in protection against various kinds of stresses (Ayaz et al., 2000; Mittler, 2006; Myung-Min et al., 2010). Phenolic compounds prevent the oxidative burst of plant cells and hence defend the plants from injury of proteins and lipids, RNA and DNA structures (Apel and Hirt, 2004). In present study water stress significantly reduced the total phenolic contents. Foliar application of growth promoters can assist plants in mitigating water stress enabling regulation of phenolic acids (Garg, 2003). Among these growth promoters, MLE produced maximum phenolic contents during both growing seasons, which protect plant tissues against oxidative damage under water stress (Ali and Ghada, 2014).

The capability of cotton plants to endure water stress depends on the production of the antioxidant enzymes such as SOD, CAT and POD (Alexieva et al., 2001). In present investigation the activity of enzymes (SOD, POD and CAT) improved considerably in water stressed environments. Whereas minimum SOD, CAT and POD were observed under well-watered conditions (Zhang et al., 2007; Ahmadizadeh et al., 2011). Foliar spray of MLE and salicylic in water stress situations significantly increased the production of antioxidants enzymes. Foliar spray of salicylic acid on water stressed plants considerably reduced the relative cell injury percentage and improved the antioxidants enzyme activities (SOD, POD, CAT and phenolic contents) therefore; enhancing the tolerance to stressed conditions (Hayat et al., 2008). Likewise, MLE being rich source of growth promoting hormones improved the production of antioxidant activities. Activation of self-defence system by exogenous application of MLE is also associated with higher mineral contents present in moringa leaves making it excellent plant growth promoter (Yasmeen et al., 2013).

\section{Conclusions}

Water stress significantly reduced the cotton productivity. This decline in productivity was the consequence of reduced sympodial branches, bolls harvested and mean boll weight that was associated with impaired morphological, physiological and biochemical characteristics of cotton plants. Exogenous application of plant growth promoters particularly salicylic acid and moringa leaf extract modulated the water stress induced losses to cotton plants. These growth promoters also enhance the antioxidant enzymes (SOD, POD and CAT, phenolic contents) activities, reduced relative cell injury percentage and improved cell membrane thermostability of cotton plants under water stress conditions.

\section{References}

Ahmadizadeh M, Nori A, Shahbazi H, Habibpour M, 2011. Effects of drought stress on some agronomic and morphological traits of durum wheat (Triticum durum) landraces under greenhouse condition. Afr. J. Biotechnol. 10:14097-107.

Akbar M, Hussain SB, 2019. Assessment of drought tolerant cotton genotypes based on seedling and physiological attributes at different moisture levels. Pure Appl. Bio. 8:93-107.

Alexieva V, Sergiev I, Mapelli S, Karanov E, 2001. The effect of drought and ultraviolet radiation on growth and stress markers in pea and wheat. Plant Cell Environ. 24:1337-44.

Ali EMA, Ghada SMI, 2014. Tomato fruit quality as influenced by salinity and nitric oxide. Turk. J. Bot. 38:122-9.

Anjum NA, Gill SS, Gill R, 2014. Plant adaptation to environmental change: significance of amino acids and their derivatives, $1^{\text {st }}$ Edn. CABI, Wallingford.

Apel K, Hirt H, 2004 Reactive oxygen species: metabolism, oxidative stress and signal transduction. Annu. Rev. Plant Biol. 55:373-99.

Arfan M, Athar HR, Ashraf M, 2007 Does exogenous application of salicylic acid through the rooting medium modulate growth and photosynthetic capacity in two differently adapted spring 
wheat cultivars under salt stress? J. Plant. Physiol. 164:685-94. Arif M, Kareem SHS, Ahmad NS, Hussain N, Yasmeen A, Anwar A, Naz S, Iqbal J, Shah GA, Ansar M, 2019. Exogenously applied bio-stimulant and synthetic fertilizers to improve the growth, yield and fiber quality of cotton. Sustainability. 11:1-14.

Ayaz FA, Kadioglu A, Turgut R, 2000. Water stress effects on the content of low molecular weight carbohydrates and phenolic acids in Ctenanthe setosa (Rosc.) Eichler. Can. J. Plant Sci. 80:373-8.

Bibi AC, Oosterhuis DM, Gonias ED, 2010. Exogenous application of putrescine ameliorates the effect of high temperature in Gossypium hirsutum L. flowers and fruit development. J. Agron. Crop. Sci. 196:205-11.

Blaise D, Singh J, Bonde A, 2009. Response of rainfed cotton (Gossypium hirsutum) to foliar application of potassium. Indian J. Agron. 54:444.

Borra's L, Westgate ME, Otegui ME, 2003. Control of kernel weight and kernel water relation by post-flowering source-sink ratio in maize. Ann. Bot. 91:857-67.

Chance M, Maehly AC, 1955. Assay of catalases and peroxidases. Meth Enzmol. 2:764.

Clarke SM, Cristescu SM, Miersch O, Harren FJ, Wasternack C, Mur LA, 2009. Jasmonates act with salicylic acid to confer basal thermotolerance in Arabidopsis thaliana. New Phytol. 182:175-87.

Farooq M, Wahid A, Kobayashi N, Fujita D, Basra SMA, 2009. Plant drought stress: effects, mechanisms and management. Agron. Sustain. Dev. 29:185-212.

Garg BK, 2003. Nutrient uptake and management under drought: nutrient moisture interaction. Curr. Agric. 27:1-8.

Giannopolitis CN, Reis SK, 1997. Superoxide dismutase I. Occurrence in higher plants. Plant Physiol. 59:309-14.

Golldack D, Li C, Mohan H, Probst N, 2014. Tolerance to drought and salt stress in plants: Unraveling the signaling networks. Front. Plant Sci. 5.

Güllüoglu L, 2004 Determination of usage of plant growth regulators in soybean (Glycine max) farming under Harran plain conditions. University of Harran (Turkey). J. Faculty Agric. 8:17-23.

Hamada A, 2000, Amelioration of drought stress by ascorbic acid, thiamin or aspirin in wheat plants. Indian J. Plant Physiol. 5:358-63.

Hayat S, Hasan SA, Fariduddin Q, Ahmad A, 2008. Growth of tomato (Lycopersicon esculentum) in response to salicylic acid under water stress. J. Plant Int. 3: 297-304.

Ismail H, Maksimovic JD, Maksimovic V, Shabala L, Živanovic BD, Tian Y, Jacobsen SE, Shabala S, 2016. Rutin, a flavonoid with antioxidant activity, improves plant salinity tolerance by regulating $\mathrm{K}+$ retention and $\mathrm{Na}+$ exclusion from leaf mesophyll in quinoa and broad beans. Funct. Plant Biol. 43:75-86.

Isokpehi RD, Mahmud O, Mbah AN, Simmons SS, Avelar L, Rajnarayanan RV, Udensi UK, Ayensu WK, Cohly HH, Brown $\mathrm{SD}, 2011$. Developmental regulation of genes encoding universal stress proteins in Schistosoma mansoni. Gene Regul. Sys. Biol. 5:61.

Kawakami EM, Oosterhuis DM, Snider JL, 2010. Physiological effects of 1- methylcyclopropene on well-watered and waterstressed cotton plants. J. Plant Growth Regul. 29:280-8.

Khan MIR, Asgher M, Khan NA, 2014. Alleviation of salt-induced photosynthesis and growth inhibition by salicylic acid involves glycinebetaine and ethylene in mungbean (Vigna radiata L.). Plant Physiol. Biochem. 80:67-74.

Korres NE, Norsworthy JK, Tehranchian P, Gitsopoulos TK, Loka DA, Oosterhuis DM, 2016. Cultivars to face climate change effects on crops and weeds: A review. Agr. Sust. Devel. 36:12.

Mittler R, 2006. Abiotic stress, the field environment and stress condition. Trends in Plant Sci. 11:15-9.

Muscolo A, Sidari M, Anastasi U, Santonoceto C, Maggio A, 2014. Effect of PEG-induced drought stress on seed germination of four lentil genotypes. J. Plant Interact. 9:354-63.

Myung-Min O, Edward EC, Rajashakar CB, 2010. Regulated water deficit improve phytochemical concentration in lettuce. Am. Soc. Hort. Sci. 135:223-9.

Noreen S, Zafar ZU, Hussain K, Athar HUR, Ashraf M, 2015. Assessment of economic benefits of foliarly applied osmoproctectants in alleviating the adverse effects of water stress on growth and yield of cotton (Gossypium hirsutum L.). Pak. J. Bot. 47:2223-30.

Rady MM, Mohamed GF, 2015. Modulation of salt stress effects on the growth, physio-chemical attributes and yields of Phaseolus vulgaris L. plants by the combined application of salicylic acid and Moringa oleifera leaf extract. Sci. Hort. 193:105-13.

Ratnakumari S, Subbaramamma P, 2006. Genetic evaluation of Gossypium hirsutum genotypes for yield, drought parameters and fibre quality. J. Cotton Res. Dev. 20:166-70.

Rehman HU, Kamran M, Basra SMA, Afzal I, Farooq M, 2015. Influence of seed priming on the performance and water productivity of direct seeded rice in alternate wetting and drying. Rice Sci. 22:189-96.

Ren X, Zhang L, Du M, Evers JB, Werf WVD, Tian X, Li Z, 2013. Managing mepiquat chloride and plant density for optimal yield and quality of cotton. Field Crops Res.149:1-10.

Sadak MS, Dawood MG, 2014. Role of ascorbic acid and $\alpha$ tocopherol in alleviating salinity stress on flax plant (Linum usitatissimum L.). J. Stress Physiol. Biochem. 10:93-111.

Sahito A, Baloch ZA, Mahar A, Otho SA, Kalhoro SA, Ali A, Kalhoro FA, Soomro RN, Ali F, 2015. Effect of water stress on the growth and yield of cotton crop (Gossypium hirsutum L.). Am. J. Plant Sci. 6:1027-39.

Saifuddin M, Osman N, Idris RM, Halim A, The effect of Pre-aluminium treatment on morphology and physiology of potential acidic slope plants. Kuwait J. Sci. 43:199-220.

Sánchez-Rodríguez E, Rubio-Wilhelmi M, 2010. Genotypic differences in some physiological parameters symptomatic for oxidative stress under moderate drought in tomato plants. J. Plant Sci. 178: 30-40.

Shan C, Liang Z, 2010. Jasmonic acid regulates ascorbate and glutathione metabolism in Agropyron cristatum leaves under water stress. Plant Sci. 178:130-9.

Steel RGD, Torrie JH, Deekey DA, 1997. Principles and procedures of statistics. A biometrical approach. $3^{\text {rd }}$ ED. Mc Graw Hill Book. Int. Co., New York, pp 400-428.

Sullivan CY, 1972. Mechanisms of heat and drought resistance in grain sorghum and methods of measurement. Sorghum in the Seventies. Oxford \& IBH Publishing Co. Pvt. Ltd., New Delhi, pp 247-284.

Veesar NF, Baloch MJ, Kumbher MB, Chachar QD, 2018. Field screening of cotton genotypes for drought tolerance on the basis of yield and fibre traits. Sindh Univ. Res. J. 50:45-52.

Waterhouse AL, 2001. Determination of total phenolics. In: R. E. Wrolstad (Ed.), Current protocols in food analytical chemistry. John Wiley and Sons, New York, NY, 11:1-8.

Wutipraditkul N, Wongwean P, Buaboocha T, 2015. Alleviation of salt-induced oxidative stress in rice seedlings by proline and/or glycinebetaine. Biol. Plant. 59:547-53.

Yasmeen A, Arif M, Hussain N, Malik W, Qadir I, 2016. 
Morphological, growth and yield response of cotton to exogenous application of natural growth promoter and synthetic growth retardant. Int. J. Agric. Biol. 18:1109-121.

Yasmeen A, Arif M, Hussain N, Naz S, Anwar A, 2018. Economic analyses of sole and combined foliar application of moringa leaf extract (MLE) and $\mathrm{k}$ in growth and yield improvement of cotton. Int. J. Agric. Biol. 20:857-63.

Yasmeen A, Basra SMA, Farooq M, Rehman HU, Hussain N, 2013. Exogenous application of moringa leaf extract modulates the antioxidant enzyme system to improve wheat performance under saline conditions. Plant Growth Regul. 69:22533.

Yosefi M, Sharafzadeh S, Bazrafshan F, Zare M, Amiri A, 2018.
Application of jasmonic acid can mitigate water deficit stress in cotton through yield-related physiological properties. Acta Agrobot. 71:1741.

Yoshida Y, Sano R, Wada T, Takabayashi J, Okada K, 2009. Jasmonic acid control of GLABRA3 links inducible defence and trichome patterning in Arabidopsis. Development. 136: 1039-48.

Zhang X, Ervin EH, 2004. Cytokinin-containing seaweed and humic acid extracts associated with creeping bentgrass leaf cytokinins and drought resistance. Crop Sci. 44:1737-45.

Zhang XC, Wu X, Findley S, Wan J, Libault M, Nguyen HT, Cannon SB, Stacey G, 2007. Molecular evolution of LysM type receptor-like kinases in plants. Plant Physiol. 144:623-36. 